\title{
Fault Detection and Classification for Transmission Line Protection System Using Artificial Neural Network
}

\author{
Ei Phyo Thwe, Min Min Oo
}

Dept. of Electrical Power Engineering, Mandalay Technological University, Mandalay, Myanmar

Email address:

email1@eiphyo2012@gmail.com (E.P.Thwe), emai12@minminooep@gmail.com (M. M. Oo)

\section{To cite this article:}

Ei Phyo Thwe, Min Min Oo. Fault Detection and Classification for Transmission Line Protection System Using Artificial Neural Network. Journal of Electrical and Electronic Engineering. Vol. 4, No. 5, 2016, pp. 89-96. doi: 10.11648/j.jeee.20160405.11

Received: September 9, 2016; Accepted: September 23, 2016; Published: October 19, 2016

\begin{abstract}
The artificial neural network is a powerful tool for the detection of the transmission line faults due to its ability to differentiate between various patterns. This paper deals with the application of artificial neural networks (ANNs) to the fault detection and classification in high voltage transmission lines for high speed protection which can be used in digital power system protection. The three phase currents of one end are taken as inputs in the proposed scheme. The feed forward neural network along with back propagation algorithm has been employed for detection and classification of the fault for analysis of each of the three phases involved in the process. The ANN was trained and tested using various sets of field data, which was obtained from the simulation of faults at various fault scenarios (fault types, fault locations and fault resistance) of $230 \mathrm{kV}$, $193.2 \mathrm{~km}$ in length "Mansan-Shwesaryan, Mandalay Region, Myanmar" transmission line using a computer program based on MATLAB/Simulink. Simulation results confirm that the proposed method can efficiently be used for accurate fault classification on the transmission line.
\end{abstract}

Keywords: Artificial Neural Network, Fault Detection, Classification, Transmission Line

\section{Introduction}

Overhead transmission lines are parts of the main components in an electric power system and, because transmission lines are exposed to the nature, the possibility of experiencing faults on transmission lines is generally higher than that on other main components. Line faults are the most common faults because lines are exposed to the elements and there are many causes of faults. Lines faults may be triggered by lightning strokes, trees may fall across lines, fog and salt spray on dirty insulators may cause the insulator strings to flash over, and ice and snow loading may cause insulator strings to fail mechanically. When a fault occurs on an electrical transmission line, it is very important to detect it and find its type and location in order to make necessary repairs and to restore power as soon as possible, the time needed to determine the fault point along the line will affect the quality of power delivery. Therefore, an accurate fault type classification and location on the line is an important requirement for a permanent fault.

In transmission line protection, the analysis of voltages and currents at the relay location is essential for the correct decision of the relay tripping action. During the fault, transients arise in both voltage and current waveforms. The past techniques used to analyze the symmetrical components of these waveforms. Some of them such as representing transmission lines by either first or second order differential equations and traveling-wave techniques have resulted in several commercial developments. However, both these approaches are based on deterministic computations on a well-defined model of the system to be protected. This results in difficulty in taking system variation into account as the rules are fixed. They do not have the ability to adapt dynamically to the system operating conditions, and to make correct decisions if the signals are uncertain.

With the advent of microprocessors and digital electronics, digital-based relaying has been developed. Research activity has covered virtually every protection technique. Furthermore, many novel algorithms and associated hardware implementations have emerged. Recently artificial intelligent techniques are used for the waveforms analysis. The artificial neural network in conjunction with wavelet transform is widely used in fault detection and classification. Various applications of neural networks were used in the past 
to improve recognition of the faults on transmission lines. The main aim of this paper is to design, develop, test and implement a complete strategy for the fault detection and classification.

Kim, et al. [1] has used Wavelet Transforms to detect the high impedance arcing faults. Jiang, et al. [2] have used Haar Wavelet to detect dc component for identifying the faulty phases. Distance protection schemes using WT based phasor estimation are reported in [3, 4]. Wavelet multi resolution analysis for location of faults on transmission lines is reported in [5]. A powerful high speed traveling wave-based protection technique using wavelet/ PCA analysis is proposed in [6]. Artificial Neural Networks (ANN) with their excellent pattern recognition capabilities, are used to estimate the fault location [7, 8]. Silva et al. [9] have Wavelet Transform and ANN for detection and classification of faults in power transmission lines. Computer based relaying system for power system has been reported in [10].

In this paper simulation of the used power system is performed using MATLAB/Simulink. Simulation outputs of large number of scenarios including various faults and locations were used as database for the analysis procedure. The current waveforms obtained from the simulation were analyzed using wavelet transform and artificial neural network. In order to minimize the size and time for training of the neural network, preprocessing of these waveforms was performed initially.

\section{Transmission Line Protection}

Fault is an unwanted short circuit condition that occurs either between two phases of wires or between a phase of wire and ground. Short circuit is the most risky fault type as low of heavy currents can cause overheating or create mechanical forces which may damage equipment and other elements of power system. Faults also can be classified into three types, such as symmetrical faults, unsymmetrical faults, and open circuit faults.

The fault that results in symmetrical fault currents (i.e., equal currents with 120 displacements) is known as a symmetrical fault. Three-phase fault is an example of symmetrical fault where all three phases are short circuited with or without involving the ground. Examples of different unsymmetrical faults are single phase to ground, two phases to ground, and phase to phase short circuits. The details of these shunt fault types that can occur in transmission line are described as follows [11].

(1) Single Phase to Ground (L-G) Fault. L-G is a short circuit between any one of phase conductors and earth (prevalence is $70 \%-80 \%$ ). It may be caused either by insulation failure between a phase conductor and earth or breaking and falling of phase conductor to the ground.

(2) Two Phases to Ground (L-L-G) Fault. L-L-G is a short circuit between any two phases and earth (prevalence is $10 \%-17 \%$ ).

(3) Phase to Phase (L-L) Fault. L-L is a short circuit between any two phases of the system (prevalence is $8 \%-10 \%)$.

(4) Three-Phase (L-L-L) Fault. L-L-L is a short circuit between any two phases of the system (prevalence is $2 \%-3 \%$ ).

When a fault occurs, the power transmission line can be protected by fuses, over current relays, distance relays, pilot protection schemes or by a combination of these relays. Fuses and over current relays are generally used for the protection of distribution lines because these devices are simple and inexpensive. Over current relays are also applicable, where a large impedance component such as a transformer, is involved. Currents due to a fault on the load end of a transformer are considerably less than the currents due to a fault on the source end. Therefore it is possible to current grade the relays. With the passage of time and increase in demand of electric energy, power systems have grown to large areas. The HV and EHV transmission lines connect the generating sources and load points located at long distance. Due to the long lengths of transmission lines and networking of the transmission systems, over current relays cannot be used to protect. Distance relays are the most often used to protect transmission lines. Distance relays take voltages and currents as inputs from the power system and calculate impedance. If the calculated impedance lies in a pre-defined operating region on the impedance plane, distance relays operate to isolate the faulted part.

Digital relays, used in the transmission line protection, are based on the same protection logic as is used in analog relays but, the physical structure and operation of the digital relays are different. Most digital relays, presently in practice, use the fundamental frequency components of the voltages and currents to protect the transmission line. Voltages and currents on a transmission line contain high frequency components at the occurrence of a fault. These components, generated by faults in the currents are shown in Figure 1. The high frequency components contain traveling waves, which originate at the fault and travel away from it. The information contained in the waves can be extracted and used to detect and locate faults. In this paper, the digital techniques which use the wavelet theory to detect and classify faults on transmission lines are proposed [12].

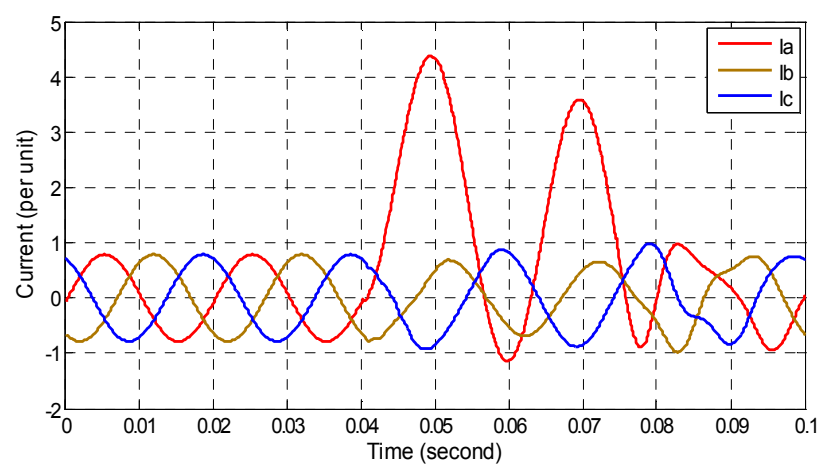

Figure 1. Three phase current waveforms in steady state, during a fault and after a fault on phase-a. 


\section{Wavelet Transform}

To improve the reliability of the protection algorithms, signal processing was introduced into transmission line protection. For instance, Wavelet Transform (WT) has been applied to extract transient characteristics of different fault conditions for analyzing various transient voltage traveling waves. WT has the function of filtering, which can get rid of noise disturbance. A technique similar to Discrete Fourier Transform (DFT) is presented for decomposing the transient components of current signals. Due to the effectiveness of WT in transient analysis, this technique has been combined with other techniques with the aim of improving the reliability of protection schemes. In other words, wavelet entropy can identify the power signals with different complexity. Therefore, the wavelet energy is applied to describe the signals with different complexity which can distinguish internal fault from external fault. Moreover, wavelet energy entropy has a unique sensibility to slight change of signals and it can reflect the energy distribution information in both time and frequency domain. The relative energy entropy is considered to be a measure of the normal signals and the faulty signals, which can discriminate between the faulty groups and the normal groups [13].The application of wavelet transform in engineering areas usually requires discrete wavelet transform (DWT).The representation of DWT can be written as:

$$
D W T(m, n)=\frac{1}{\sqrt{a_{0}^{m}}} \sum_{k} x(k) \varphi\left(\frac{k-n b_{0} a_{0}^{m}}{a_{0}^{m}}\right)
$$

where $a_{0}$, is the scale factor and $b_{0}$ is the translation factor,.the functions of integers ' $m$ ', ' $n$ '. ' $k$ ' is an integer variable and it refers to a sample number in an input signal. A very useful implementation of DWT, called multi-resolution analysis, is demonstrated in Figure 2.

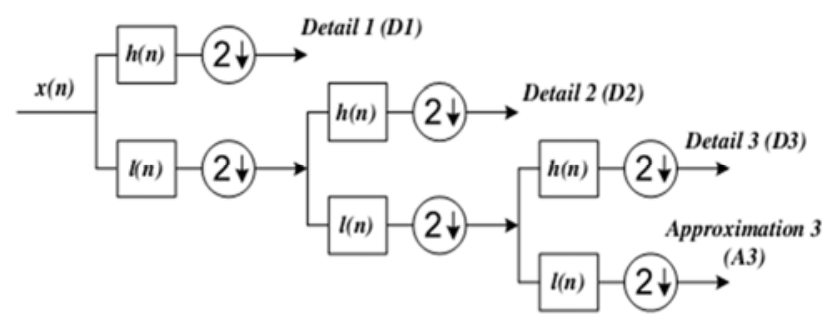

Figure 2. Wavelet multi-resolution analysis.

The original sampled signal $\mathrm{x}(\mathrm{n})$ is passed through a high pass filter $\mathrm{h}(\mathrm{n})$ and a low pass filter $1(\mathrm{n})$. Then the outputs from both filters are decimated by 2 to obtain the detail coefficients and the approximation coefficients at level 1 (D1 and A1). The approximation coefficients are then sent to the second stage to repeat the procedure. Finally, the signal is decomposed at the expected level [14]. In the case shown in Figure 2, if the original sampling frequency is $\mathrm{F}$, the signal information captured by D1 is between $F / 4$ and $F / 2$ of the frequency band. D2 captures the information between $\mathrm{F} / 8$ and F/4. D3 captures the information between F/16 and F/8, and $\mathrm{A} 3$ retains the rest of the information of original signal between 0 and $F / 16$. By such means, useful information can be easily extracted from the original signal into different frequency bands and at the same time the information is matched to the related time period. The original signal is one cycle of a post-fault current signal, as shown in Figure 3. Db4 wavelet is used to make a 5 level decomposition. The reconstructed versions of each detail and the approximation are shown in Figure 3. The information of original signal is clearly represented at each frequency band. The original signal can be reconstructed by adding up those wavelet signals at the same sample point. The wavelet toolbox in MATLAB provides a lot of useful techniques for wavelet analysis.

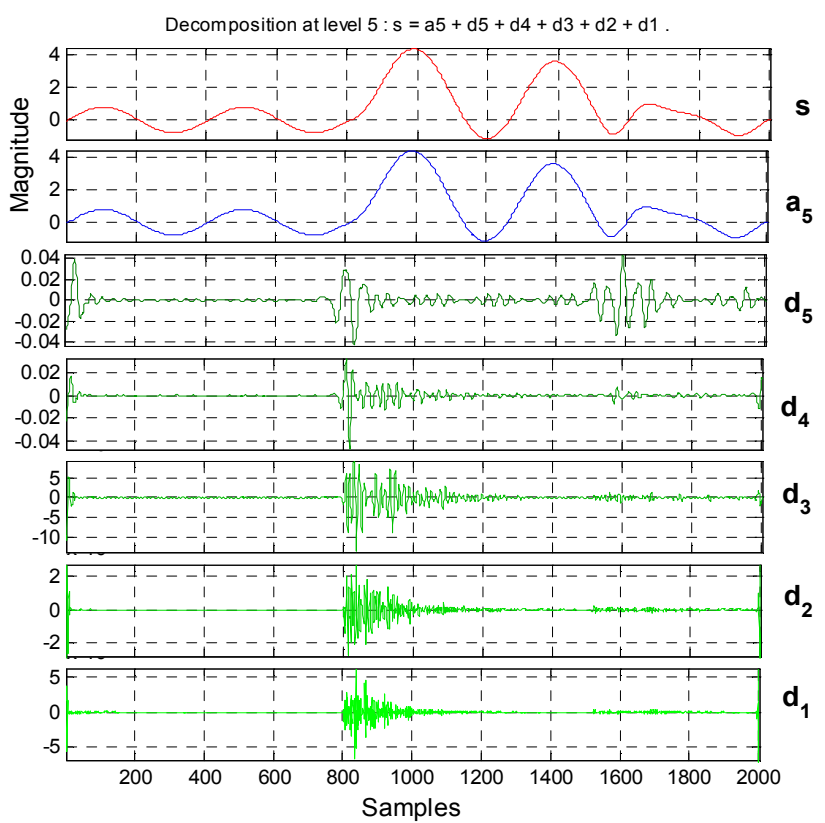

Figure 3. An example of wavelet multi-resolution analysis.

\section{Spectral Energy from Wavelet Transform}

It can be seen from Figure 3 that the wavelet coefficients $\mathrm{d} 1$ to $\mathrm{d} 5$ around the fault inception point fluctuate between positive and negative values. Although, they are quite distinctive features for fault identification, they are not discernable enough to be directly employed in the decision making process about a particular fault; hence the spectral energy is firstly extracted from these signals and it can be calculated using the following formula:

$$
E_{j}(k)=\sum_{n=20(k-1)+1}^{20 k} c D_{j}(n)^{2}, k=1,2,3 \ldots
$$

where $\mathrm{E}$ is the spectral energy, ' $\mathrm{n}$ ' is the coefficient number, ' $\mathrm{k}$ ' is the window's number, ' $\mathrm{j}$ ' is the wavelet decomposition level and $\mathrm{cD}$ is the magnitude of the coefficient for the details from WT. Each spectral energy data contains the energy during a certain window length. In this paper, the number of 
one coefficient is selected. The reason is that too less window length will impact on the accuracy. Based on the coefficients generated from the MATLAB wavelet toolbox, the spectral energy can be calculated and utilized using equation 2 .

\section{Artificial Neural Network}

Artificial neural network (ANN) has been equipped with distinctiveness of parallel processing, nonlinear mapping, associative memory, and offline and online learning abilities. The wide uses of ANN with its conquering outcomes make it an effective diagnostic mean in electric power systems. Its versatility with multitude applicability can be seen in other areas of science and engineering research.

ANN is trained by using prior chosen fault samples as input and set of fault information as output for fault diagnosis application [15]. Neural networks are comprised of primarily three basic learning algorithms such as supervised learning, unsupervised learning, and reinforced learning. Among these supervised learning is most commonly used and is also referred to as learning with a teacher. This is applied when the target is having identified value and is associated with each input in the training set. Figure 4 represents the supervised architecture of ANN.

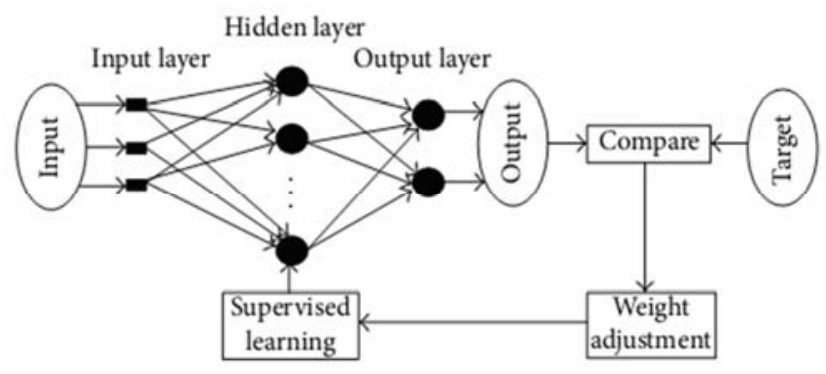

Figure 4. Supervised architecture of ANN.

Error back propagation (BP) neural network was applied for diagnosis of fault in power system. However slow speed training and the shortcomings of local optimal lead to the introduction of additional momentum factor for problem solving. Radial basis function (RBF) neural network has a faster learning speed and the ability of arbitrary function approximation. In this paper, (RBF) is employed for fault detection and classification for transmission line protection system.

Artificial neural network (ANN) can be applied to fault detection and classification effectively because it is a programming technique, capable to solve the nonlinear problems easily. The ANNs are able to learn with experiences. They are widely accepted and used in the problem of fault detection and fault classification because of the following features:

- Number of transmission line configuration are possible as there can be any possibility from short length, long length, single circuit transmission line to double-circuit transmission lines, etc.

- There are several methods to simulate the network with different power system conditions in a fast and reliable manner.

- The conditions of the electrical power system change after each and every disturbance. Hence a neural network is capable to incorporate the dynamic changes in the power systems.

- The ANN output is very fast, reliable and accurate depending on the training, because its working depends upon a series of very simple operations.

The algorithm which employed ANNs programming offers many advantages, but it also suffers with many disadvantages, which are very complex in nature. Some of the important factors are the selection of type of network, architecture of the network (which includes the selection of number of layers, number of neurons in each layer, selection of activation functions, learning algorithms parameters etc.), termination criteria etc. There are various parameters like values of the pre fault and post fault voltages and currents of the respective three phases in steady state required for precise fault detection and classification.

The values of the pre fault and post fault voltage and current of respective three phases are very different and are governed by the type of fault. Thus, the fault classification method required a neural network that allows it to determine the type of fault from the patterns of pre fault and post fault voltages and currents, which are generated from the values measured from a three phase transmission line of an electrical power system at one terminal. The neural network is based upon the total three numbers of inputs, i.e. the currents of respective three phases. The neural network is trained by using these six inputs. The total number of outputs of the neural network is four in numbers, i.e. three phases A, $\mathrm{B}, \mathrm{C}$ and fourth is ground of three phase transmission line [16].

\section{Fault Classification by Ann}

Although the basic concept behind relays remains the same, the digital technology has had a significant influence on the way relays operate and have offered several improvements over traditional electromechanical relays. In ANN based method, the entire data that is collected is subdivided into three sets namely the training, validation and the testing data sets. The first step in the process is fault detection. Once we know that a fault has occurred on the transmission line, the next step is to classify the fault into the different categories based on the phases that are faulted. The aim of this paper is to propose an integrated method to perform each of these tasks using artificial neural networks. A back-propagation based neural network has been used for the purpose of fault detection and fault classification. For each of the different kinds of faults, separate neural networks have been employed for the purpose of fault classification. Each of these steps has been depicted in the flowchart shown in Figure 5.

The flowchart explains the basic procedure which was used to implement the ANN based fault detection and 
classification in HV transmission line. During Preprocessing, three current input signals were sampled at a sampling frequency of 1.0 MH and further processed by lowpass filter. Then one full cycle Discrete Fourier Transform is used to calculate the energy output of three currents. The input signals were normalized in order to make the ANN input level between +1 or 0 . The most important aspects of the fault location systems are network architecture, selection of learning rule, training method, and testing the fault locator based on ANN.

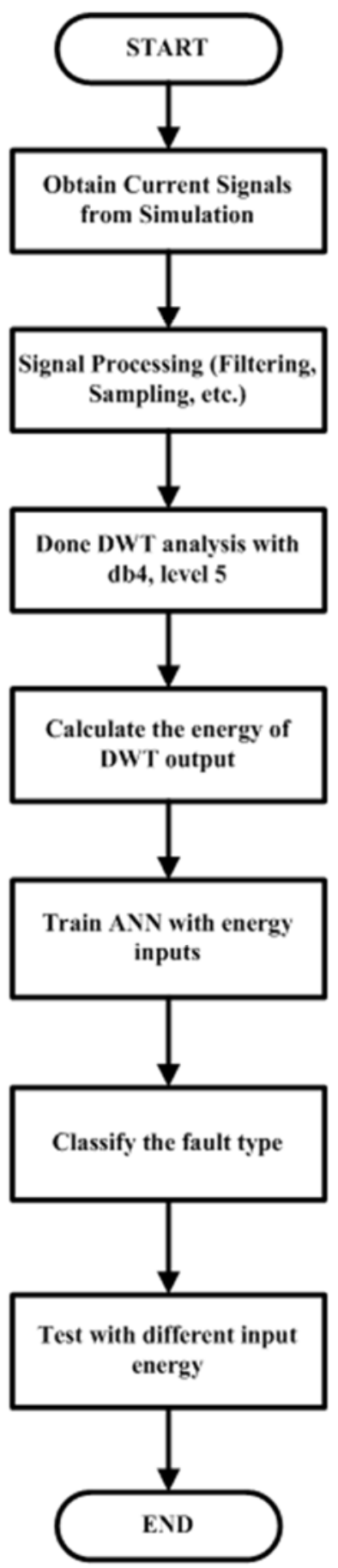

Fault Classification

Figure 5. Flowchart depicting the outline of ANN Based.

\section{Execution for Ann Based Fault Classification}

For the ANN based fault detection and classification, the detailed study is executed at "Mansan-Shwesaryan, Mandalay Region, Myanmar" $230 \mathrm{kV}$ twin bundled double circuit transmission line. The Simulink model for the system under study is "Mansan-Shwesaryan, Mandalay Region, Myanmar" $230 \mathrm{kV}$ twin bundled double circuit transmission line shown in Figure 6. It consists of $230 \mathrm{kV}$ transmission line $193.2 \mathrm{~km}$ in length. A source is located at Mansan bus and a load is attached to Shwesaryan bus. The transmission lines are represented by distributed parameter lines. The entire transmission line is divided into two and a fault is located between them as shown in Figure.

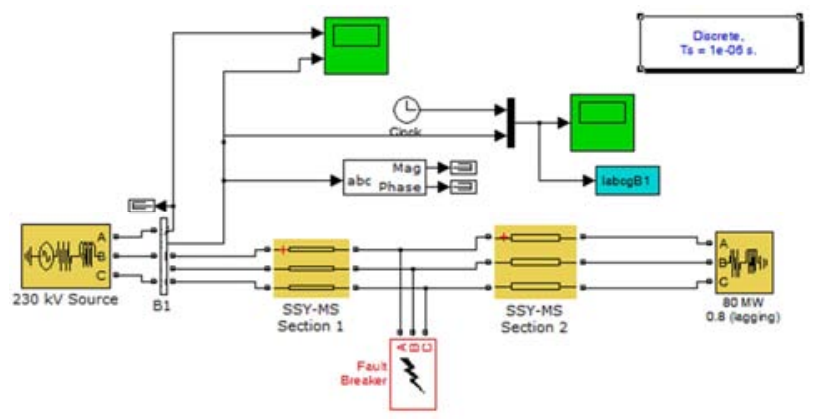

Figure 6. Simulink Model for ANN Based Fault Detection and Classification.

The important parameters for the model are obtained from line characteristics and conductor size. They are as follow:

Line Length $=120$ mile $(193.2 \mathrm{~km})$

Positive/negative Sequence Impedance $=0.053+\mathrm{j} 0.142 \mathrm{ohm} / \mathrm{km}$

Zero Sequence Impedance $=0.2856+\mathrm{j} 0.7205 \mathrm{ohm} / \mathrm{km}$

Positive/ negative Sequence Susceptance $=7.92 \mu \mathrm{S} / \mathrm{km}$

Zero Sequence Susceptance $=3.84 \mu \mathrm{S} / \mathrm{km}$

Fault Starting $=0.05$ seconds

Duration in fault $=0.03$ Seconds

Type Conductor $=$ Peacock

Simulation time is set as 0.1 second with sampling time of $1 \mu \mathrm{sec}$. Thus sampling frequency of the model is $1 \mathrm{MHz}$.

In the model, the current measurements are carried out at Mansan bus (B1) and these values are used for fault classification. The current values are saved at workspace in .mat format and then converted to. csv files. Based on the. csv files, the spectral energy for each current is obtained from matlab program simulations. To obtain different spectral energy, ten types of faults are performed with different fault resistance and location as shown in Table 1.

Table 1. Input Data for ANN Training.

\begin{tabular}{ll}
\hline Parameter & Set Type \\
\hline \multirow{2}{*}{ Fault Type } & $\mathrm{AG}, \mathrm{BG}, \mathrm{CG}, \mathrm{ABG}, \mathrm{BCG}, \mathrm{ACG}, \mathrm{AB}, \mathrm{BC}, \mathrm{AC}$, \\
Fault Location in & $\mathrm{ABC} ;$ \\
percent & $70,10,15,20,25,30,35,40,45,50,55,60,65$, \\
Fault Resistance $(\Omega)$ & $0.05,0.1,0.2,0.5,1,2,5,10,20,50 ;$ \\
\hline
\end{tabular}


According to Table 1, 1900 input training data are obtained for ANN based fault classification. There are four outputs from ANN as binary digit. Then $(1900 \times 3)$ input data and $(1900 \times 4)$ target data are fed to 'nprtool' (Neural network pattern-recognition tool). From 400 input data, $70 \%$ are used for training, $20 \%$ for validation and 10 percent for testing. In the ANN training, 2 layers with 50 neurons are set as the basic parameters. The weight and bias are set random according to default toolbox parameter. The sample input and target data for AG fault with $20 \Omega$ fault resistance with various fault distances are depicted in Table 2. For the training case, the Neural Network structure is shown in Figure 7 and performance curve is shown in Figure 8.

Table 2. Input and Target Data for AG Fault with $20 \Omega$ Fault Resistance with Various Fault Distances.

\begin{tabular}{llllll|l|lll}
\hline Fault & Distance & \multicolumn{7}{l}{ Input (Energy) } & \multicolumn{3}{l}{ Output } \\
\cline { 3 - 9 } Type & $\mathbf{( \% )}$ & Ea & Eb & Ec & A & B & C & G \\
\hline AG & 5.0 & 54.1277 & 120.7274 & 116.9482 & 1 & 0 & 0 & 1 \\
AG & 10 & 52.0354 & 118.0951 & 114.3549 & 1 & 0 & 0 & 1 \\
AG & 15 & 54.0901 & 113.4338 & 109.7657 & 1 & 0 & 0 & 1 \\
AG & 20 & 52.5765 & 112.1197 & 108.4739 & 1 & 0 & 0 & 1 \\
AG & 25 & 81.2352 & 125.072 & 121.2121 & 1 & 0 & 0 & 1 \\
AG & 30 & 235.4797 & 129.7165 & 133.0255 & 1 & 0 & 0 & 1 \\
AG & 35 & 396.2904 & 151.9349 & 155.5067 & 1 & 0 & 0 & 1 \\
AG & 40 & 432.0988 & 133.0745 & 136.3948 & 1 & 0 & 0 & 1 \\
AG & 45 & 394.4423 & 128.9449 & 125.0358 & 1 & 0 & 0 & 1 \\
AG & 50 & 375.338 & 56.4228 & 53.8582 & 1 & 0 & 0 & 1 \\
AG & 55 & 266.2426 & 34.2566 & 34.1284 & 1 & 0 & 0 & 1 \\
AG & 60 & 103.6569 & 30.0762 & 32.0047 & 1 & 0 & 0 & 1 \\
AG & 65 & 154.5392 & 101.1157 & 104.6326 & & 0 & 0 & 1 \\
AG & 70 & 345.0859 & 129.9974 & 133.9842 & 1 & 0 & 0 & 1 \\
AG & 75 & 316.3685 & 101.0683 & 104.2924 & 1 & 0 & 0 & 1 \\
AG & 80 & 421.6305 & 88.7826 & 91.6339 & 1 & 0 & 0 & 1 \\
AG & 85 & 415.235 & 95.3516 & 98.2895 & 1 & 0 & 0 & 1 \\
AG & 90 & 370.0323 & 95.6632 & 98.6147 & 1 & 0 & 0 & 1 \\
AG & 95 & 301.2343 & 90.869 & 93.7549 & 1 & 0 & 0 & 1 \\
\hline
\end{tabular}

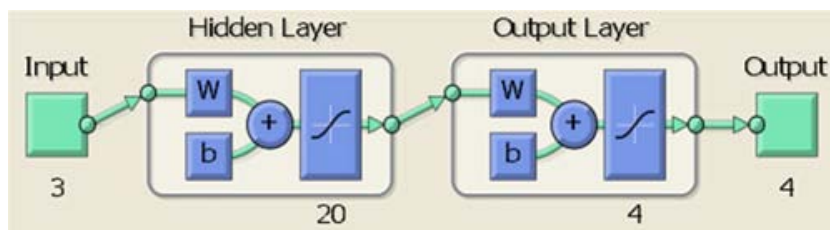

Figure 7. Neural Network Structure for Fault Classification.

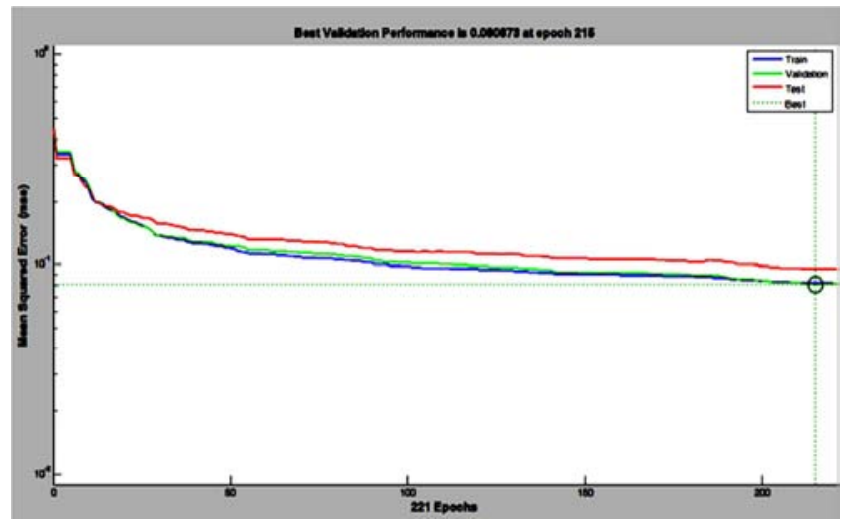

Figure 8. Performance curve for fault classification.

To obtain accurate outputs for fault classification, several trainings are carried out during simulations. Once trained, the networks performance was tested using a validation data set. The suitable network which showed satisfactory results was finally selected. When acceptable output and negligible error is obtained, Simulink model output for fault classification is generated from 'nprtool'. According to the ANN training, the resulting Simulink block for fault classification is shown in Figure 9.

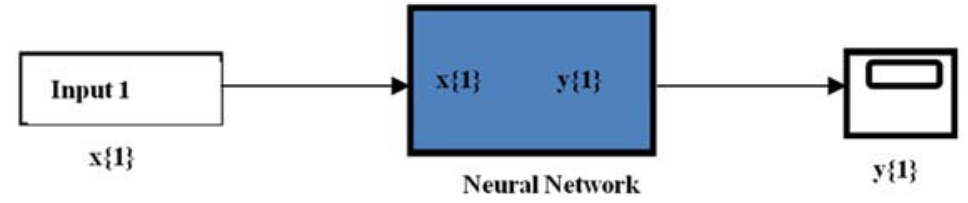

(a)

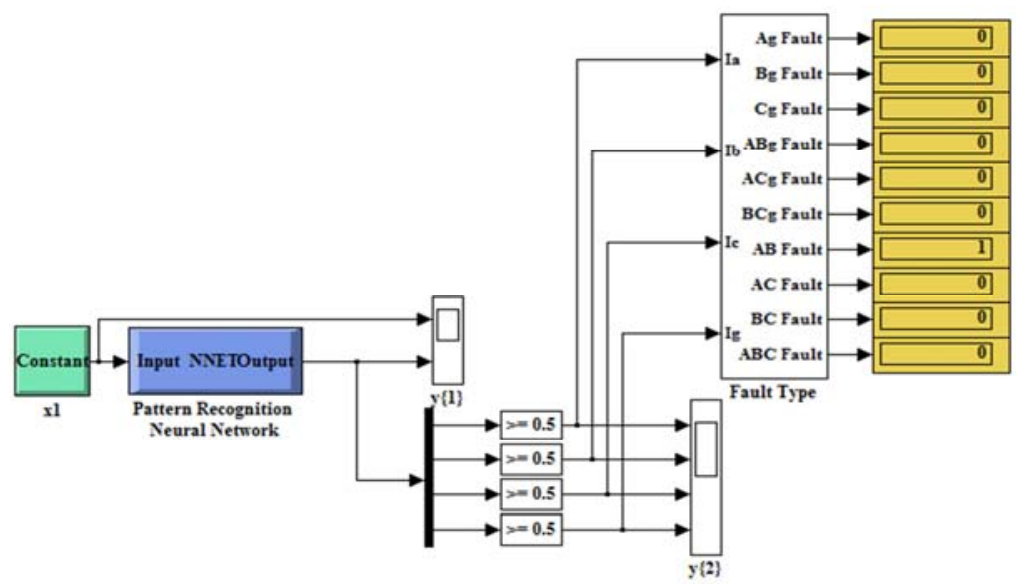

(b)

Figure 9. Simulink Block: (a) ANN Output and (b) Testing Arrangement. 


\section{Analysis on the Simulation Result}

The trained ANN based fault classifier modules were then extensively tested using independent data sets consisting of fault scenarios never used previously in training. Fault type, fault location and fault time were changed to investigate the effects of these factors on the performance of the proposed algorithm. Table 3 gives some examples for the test results.
The left four columns are the desired outputs and the right four columns are the actual outputs of the ANNs.

Simulation results for fault type classification shown in Table 3. According to the simulation results, the Neural Network application for classification of faults is consistent. In case of fault classification, ANN output display same result as desired output.

Table 3. Desired Outputs and Actual Outputs of the ANNs.

\begin{tabular}{|c|c|c|c|c|c|c|c|c|c|c|c|}
\hline \multirow{2}{*}{ SN } & \multirow{2}{*}{ Fault Resistance (ohm) } & \multirow{2}{*}{ Fault Type } & \multirow{2}{*}{ Fault Distance (\%) } & \multicolumn{4}{|c|}{ Desired Output } & \multicolumn{4}{|c|}{ ANN Output } \\
\hline & & & & $\mathbf{A}$ & B & $\mathbf{C}$ & G & $\mathbf{A}$ & B & C & G \\
\hline 1 & 2 & $\mathrm{AG}$ & 5 & 1 & 0 & 0 & 1 & 1 & 0 & 0 & 1 \\
\hline 2 & 5 & BG & 10 & 0 & 1 & 0 & 1 & 0 & 1 & 0 & 1 \\
\hline 3 & 10 & CG & 15 & 0 & 0 & 1 & 1 & 0 & 0 & 1 & 1 \\
\hline 4 & 20 & $\mathrm{ABG}$ & 20 & 1 & 1 & 0 & 1 & 1 & 1 & 0 & 1 \\
\hline 5 & 2 & BCG & 25 & 0 & 1 & 1 & 1 & 0 & 1 & 1 & 1 \\
\hline 6 & 5 & ACG & 30 & 1 & 0 & 1 & 1 & 1 & 0 & 1 & 1 \\
\hline 7 & 10 & $\mathrm{AB}$ & 35 & 1 & 1 & 0 & 0 & 1 & 1 & 0 & 0 \\
\hline 8 & 20 & $\mathrm{BC}$ & 40 & 0 & 1 & 1 & 0 & 0 & 1 & 1 & 0 \\
\hline 9 & 2 & $\mathrm{AC}$ & 50 & 1 & 0 & 1 & 0 & 1 & 0 & 1 & 0 \\
\hline 10 & 5 & $\mathrm{ABC}$ & 90 & 1 & 1 & 1 & 0 & 1 & 1 & 1 & 0 \\
\hline
\end{tabular}

with Different Scenarios

\section{Discussion and Conclusion}

A new ANN-based approach to real-time fault classification in power transmission systems which can be used in digital power system protection has been proposed in this paper. It uses calculated spectral energy as inputs. Simulation models of the transmission line system are constructed and the generated information is then channeled using MATLAB software and accompanying Power System Block Set. Various fault types (AG, BG, CG, ABG, BCG, $\mathrm{ACG}, \mathrm{AB}, \mathrm{BC}, \mathrm{AC}$, and $\mathrm{ABC})$ are detected and classified. The neural network performance of the proposed scheme is evaluated using various fault types. It was shown that the network was able to perform fast and correctly for different combinations of fault conditions, e.g. fault resistance, fault type and fault location. The fault is identified just in a few milliseconds which prove that the network is able to detect and classify the fault quite fast. All the test results show that the proposed fault detector and classifier can be used for supporting a new generation of very high speed protective relaying systems.

\section{References}

[1] Kim Chul-Hwan, Kim Hyun, Ko Young-Hun, Byun SungHyun, Aggarwal Raj K, Johns Allan T. A novel fault-detection technique of high-impedance arcing faults in transmission lines using the wavelet transform. IEEE Trans Power Delivery 2002; 17 (4): 921-9.

[2] Joe-Air Jiang, Ping-Lin Fan, Ching-Shan Chen, Chi-Shan Yu, Jin-Yi Sheu. A Fault Detection and Faulted Phase Selection Approach for Transmission Lines with Haar Wavelet Transform. In: Transmission and distribution conference and exposition
2003, vol. 3, IEEE PES, 7-12, September 2003. p. 285-9.

[3] Liang Feng, Jeyasura B. Transmission line distance protection using wavelet transform algorithm. IEEE Trans Power Delivery 2004; 19 (2): 545-53.

[4] Osman AH, Malik OP. Transmission line distance protection based on wavelet transforms. IEEE Trans Power Delivery 2004; 19 (2): 515-23.

[5] Chanda D, Kishore NK, Sinha AK. A Wavelet multiresolution analysis for location of faults on transmission lines. Electrical Power Syst Res 2003; 25: 59-69.

[6] Jafarian Peyman, Sanaye-Pasand Majid. A traveling wavebased protection technique using Wavelet/PCA analysis. IEEE Trans Power Delivery 2010; 25 (2): 588-99.

[7] Martin Fransisco, Aguado Jose A. Wavelet-based ANN approach for transmission line protection. IEEE Trans Power Delivery 2003; 18 (4): 1572-4.

[8] Tawfik MM, Marcos MM. ANN-based techniques for estimating fault location on transmission lines using prony method. IEEE Trans Power Delivery 2001; 16 (2): 219-24.

[9] Silva KM, Souza BA, Brito NSD. Fault detection and classification in transmission lines based on wavelet transform and ANN. IEEE Trans Power Delivery 2006; 21 (4): 2058-63.

[10] Phadke Arun G, Throp James S. Computer relaying for power systems. Research Study Press Ltd.; 1994.

[11] Anamika Y, Yajnaseni D, "An Overview of Transmission Line Protection by Artificial Neural Network: Fault Detection, Fault Classification, Fault Location, and Fault Direction Discrimination," vol. 2014, ID. 230382, 20 pages, 2014.

[12] Majid Jamil, Sanjeev Kumar Sharma ${ }^{*}$ and Rajveer Singh, "Fault detection and classification in electrical power transmission system using artificial neural network", Jamil et al. Springer Plus (2015). 
[13] Subba Reddy. B, D. Sreenuvasulu Reddy, Dr. G. V. Marutheswar, "Identification of Fault Location in Multiple Transmission Lines by Wavelet Transform", International Journal of Computational Engineering Research, vol. 4, issue. $2,2014$.

[14] Qais Hashim Alsafasfeh, "Pattern Recognition for Fault Detection, Classification, and Localization in Electrical Power Systems”, Ph. D Thesis, Western Michigan University, 2010.

\section{Biography}

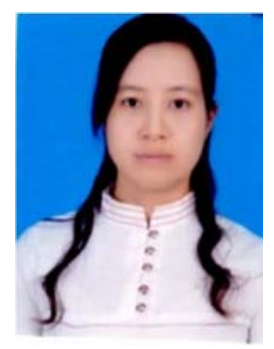

\section{Ms. Ei Phyo Thwe}

She received the BE degree in Electrical Power Engineering from Technological University (Meiktila), Myanmar in 2007 and also got her Master Degree in 2010 from the same university. Now, she is working as an Instructor at Technological University (Mandalay) Under Ministry of Science and Technology, Myanmar. Currently, she is also doing her Ph.D (thesis) in Electrical Power Engineering Department at Mandalay Technological University, Mandalay, Myanmar. Her research interests include Power System Protection, Transmission \& Distribution Systems and Artificial Intelligence.
[15] Huan, V. P., and Hung, L. K., "An ANFIS based approach to improve the fault location on $110 \mathrm{kV}$ transmission line Dak Mil - Dak Nong", IJCSI International Journal of Computer Science Issues, Vol. 11, Issue 3, No 1, 2014.

[16] Vu Phan Huan, Le Kim Hung, Nguyen Hoang Viet, "Fault Classification and Location on $220 \mathrm{kV}$ Transmission line Hoa Khanh - Hue Using Anfis Net," Journal of Automation and Control Engineering, vol. 3, no. 2, April 2015.

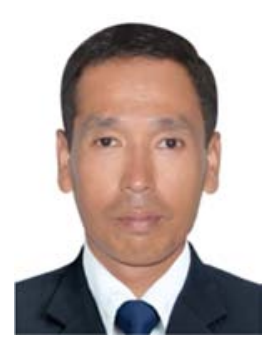

\section{Mr. Min Min Oo}

He received his BE Degree ME Degree and Ph.D Degree from Mandalay Technological University, Mandalay, Myanmar in 2000, 2002, and 2008 respectively. $\mathrm{He}$ is an Associate Professor at Department of Electrical Power Engineering, Mandalay Technological University. His research interest includes Microcontroller Based Control and Design,and Power System. 